Check for updates

Cite this: Mater. Chem. Front., 2020, 4, 950

Received 8th December 2019

Accepted 24th January 2020

DOI: 10.1039/c9qm00741e

rsc.li/frontiers-materials

\section{Pillar[5]arene-based tunable luminescent materials via supramolecular assembly-induced Förster resonance energy transfer enhancement $\dagger$}

\author{
Nan Song, ${ }^{a b}$ Xin-Yue Lou, ${ }^{a}$ Hao Yu, ${ }^{a}$ Paul S. Weiss, (D) ${ }^{c}$ Ben Zhong Tang (D) ${ }^{b}$ and \\ Ying-Wei Yang (iD *ac
}

Pillar[5]arene tetramers with tetraphenylethene cores and different lengths of alkyl ether chains (H1-4C4P and H2-2C4P) and a 9,10-distyrylanthrace-bridged neutral guest linker (DSA-G) are synthesized to fabricate tunable solid-state fluorescent materials through supramolecular assemblies of DSA-G $\subset$ H1-4C4P and DSA-G $\subset \mathbf{H 2 - 2 C 4 P}$. Their fluorescence emission is strongly enhanced and their colors are changed upon assembly, which can be ascribed to the supramolecular assembly-induced enhanced emission and Förster resonance energy transfer (FRET) processes between H1-4C4P (or H2-2C4P) and DSA-G. Both ensembles of DSA-G $\subset \mathbf{H 1}-4 C 4 P$ and DSA-G $\subset$ H2-2C4P exhibit thermo and solvent dual-responsive features, while DSA-G $\subset \mathbf{H 2}$-2C4P shows higher sensitivity toward external stimuli as compared to DSA G $\subset \mathbf{H 1 - 4 C 4 P}$ due to the shorter distance between fluorophores. The use of these fluorescent materials as inks confirms their efficiencies in the solid-state, paving the way for new potential applications of smart optical materials based on supramolecular assemblies.

\section{Introduction}

Photoluminescent materials, especially solid-state materials with tunable wavelengths, have attracted significant attention due to their great potentials in fluorescent probes and sensors, optoelectronic devices, light-emitting diodes, bioimaging agents, molecular optical devices, data storage, and security printing. ${ }^{1-3}$ However, most of their applications have been impeded because of the intrinsic self-quenching properties of most luminescent dyes. Aggregation-induced emission (AIE) $)^{4,5}$ and aggregation-induced enhanced emission (AIEE) ${ }^{6}$ have been widely developed since $2001,{ }^{7}$ which solved the drawback of self-quenching. ${ }^{8-10}$ Meanwhile, supramolecular assembly has proven to be a highly efficient way to fabricate smart luminescent materials with well-ordered architectures bearing typical

\footnotetext{
${ }^{a}$ State Key Laboratory of Inorganic Synthesis and Preparative Chemistry, International Joint Research Laboratory of Nano-Micro Architecture Chemistry, College of Chemistry, Jilin University, Changchun 130012, China.

E-mail: ywyang@jlu.edu.cn

${ }^{b}$ Department of Chemistry, HKUST Jockey Club Institute for Advanced Study Division of Life Science, Institute of Molecular Functional Materials and Division of Biomedical Engineering, The Hong Kong University of Science and Technology (HKUST), Clear Water Bay, Kowloon, Hong Kong, China

${ }^{c}$ California NanoSystems Institute and Departments of Chemistry \& Biochemistry, Bioengineering, and Materials Science \& Engineering, University of California, Los Angeles, Los Angeles, California 90095, USA

$\dagger$ Electronic supplementary information (ESI) available. See DOI: 10.1039/ c9qm00741e
}

luminophores, new physical properties, optional assembly of entities, and reversible stimuli-responsiveness. ${ }^{11-15}$ Nevertheless, luminescent materials with tunable properties adjusted via pillar $[n]$ arene-based supramolecular interactions both in solution and the solid state are less reported.

Pillar[n]arenes (pillararenes), ${ }^{16-19}$ consisting of $n$ hydroquinone units linked by methylene bridges at para positions, have contributed significantly to the development of supramolecular chemistry and materials science since the first report by Ogoshi et al. in 2008. ${ }^{16}$ As a new class of important synthetic macrocycles, ${ }^{20}$ pillararenes possess tailorable structures, facile functionality, ${ }^{21-23}$ and superior host-guest properties. ${ }^{24-29}$ A variety of pillararene-based supramolecular assemblies have been reported, which have been extensively used across a plethora of fields including molecular switches and molecular machines, ${ }^{30,31}$ metal-organic frameworks, ${ }^{32}$ controlled drugdelivery systems, ${ }^{33-38}$ artificial transmembrane channels, ${ }^{39-41}$ sensors, ${ }^{42-48}$ stimuli-responsive materials, ${ }^{49-55}$ hybrid absorbents, ${ }^{56-59}$ and biomedical applications, ${ }^{60-65}$ including as virus inhibitors and antimicrobials. ${ }^{64,65}$

Several pillararene-based fluorescent systems combined with tetraphenylethene (TPE) $)^{6-68}$ and/or 9,10-distyrylanthracene (DSA) ${ }^{69-71}$ AIEgens have also been reported for potential applications as detectors, drug-delivery systems, etc. $^{72-77}$ On the basis of our previous works, ${ }^{66,69}$ we envision that the pillararene-based supramolecular solid-state materials with tunable wavelengths can be constructed from host-guest 


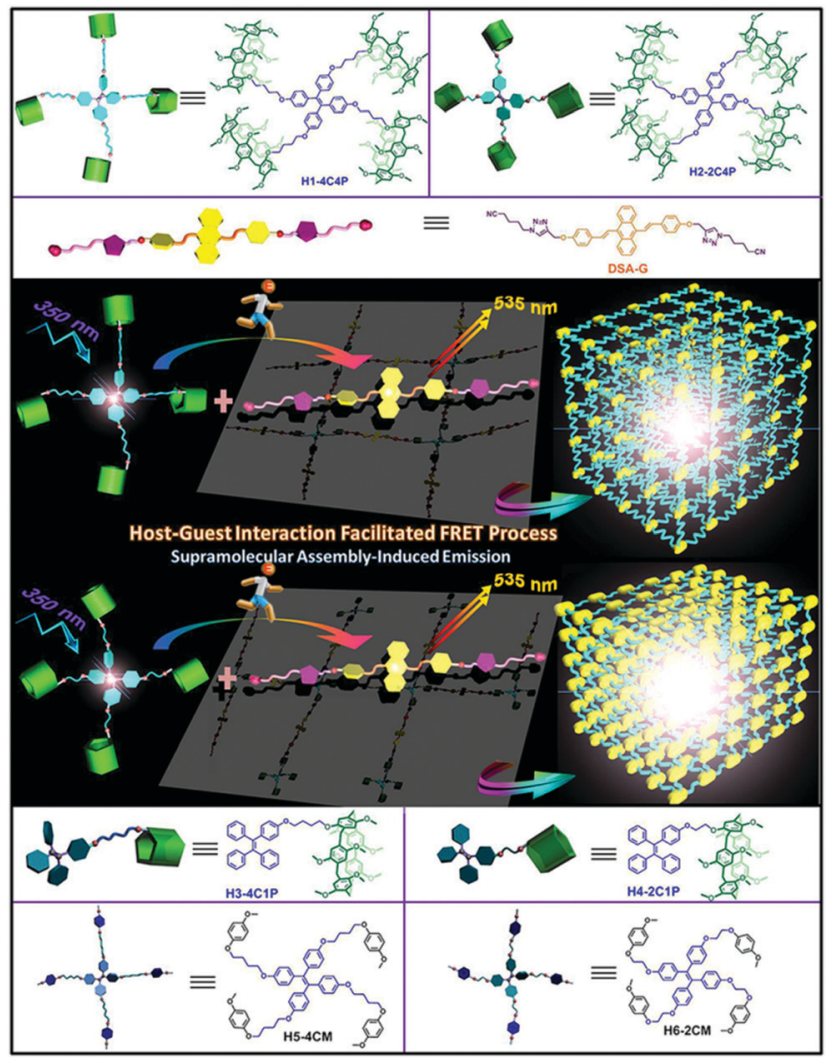

Scheme 1 Schematic representation of the Förster resonance energy transfer (FRET) process facilitated by the combination of pillararene tetramer-based host-guest complexation and supramolecular assemblyinduced emission enhancement.

complexation between TPE- and DSA-linked pillararene derivatives and guest compounds. Here, we designed and synthesized TPE-bridged pillararene tetramers and a DSA-bridged neutral guest linker for the construction of supramolecular fluorescent materials with tunable wavelengths through Förster resonance energy transfer (FRET), taking advantage of cooperative supramolecular assembly and host-guest interactions (Scheme 1).

\section{Results and discussion}

DSA-G, including two triazole-cyano binding sites bridged by the yellow AIEgen DSA, functions as the energy acceptor. ${ }^{68} \mathbf{H 2 - 2 C 4 P}$, possessing shorter alkyl chains compared with another pillararene tetramer (H1-4C4P), ${ }^{66}$ was successfully synthesized and used for comparison to demonstrate the role of chain length in host compounds. H3-4C1P, H4-2C4P, H5-4CM, and H6-2CM have also been synthesized as control compounds. All the newly synthesized compounds have been fully characterized by ${ }^{1} \mathrm{H}$ NMR, ${ }^{13} \mathrm{C} N M R$, and MALDI-TOF MS spectroscopy (Fig. S1-S14, ESI $\dagger$ ). DSA-G and H2-2C4P were endowed with AIE properties in mixed solvents, as expected (Fig. S15 and S16, ESI $\dagger$ ). The sharp quenching of fluorescence of DSA-G upon increasing the fraction of unfavourable solvents to above $80 \%$ is ascribed to sedimentation due to its poor solubility.

The pillar[5]arene ring and triazole-cyano unit can form inclusion complex with a binding affinity of $1.46 \times 10^{4} \mathrm{M}^{-1} \cdot{ }^{66}$
In order to develop their applications as supramolecular optical materials, the photophysical properties have been studied in chloroform via fluorescence spectroscopy, in which there is efficient host-guest binding affinity between the pillararene cavity and the triazole-cyano site. Ultraviolet-visible (UV-Vis) absorption spectra of H1-4C4P, DSA-G, and DSA-G $\subset$ H1-4C4P are shown in Fig. S17 (ESI $\dagger$ ). The fluorescent emission of H1-4C4P excited at $350 \mathrm{~nm}$ overlaps with the absorption spectrum of DSA-G, conforming to one of the basic principles of FRET donor-acceptor systems. Similarly, H2-2C4P can also be used to construct an optimal FRET system, upon assembly with DSA-G. As in Fig. S18 (ESI $\dagger$ ), the conformational simulation of DSA-G, H1-4C4P, and H2-2C4P with energy minimization was simply conducted, respectively. The distances from the center of TPE core to the pillar[5] arene terminals of H1-4C4P and H2-2C4P is about $14.851 \AA$ and $10.339 \AA$, respectively, while the distance from DSA core of DSA-G to the triazole-cyano binding site is about $15.294 \AA$ A. The efficient distance for FRET is $c a$. 1-10 nm, while the distances between host-guest complexes of DSA-G $\subset$ H1-4C4P and DSA-G $\subset$ H2-2C4P are $c a .3 .0 \mathrm{~nm}$ and $2.6 \mathrm{~nm}$. After the formation of supramolecular assemblies, the distance might decrease due to the twisted and interactional components, but still within the efficient distance for FRET.

Their fluorescence has been investigated in chloroform (Fig. 1a). Both H1-4C4P and H2-2C4P exhibited blue fluorescence with emission maxima at $481 \mathrm{~nm}$. After the addition of DSA-G, red-shifted fluorescence emission can be detected at $510 \mathrm{~nm}$. Upon continuous addition of DSA-G to the solution, their emission maxima underwent a $\sim 25 \mathrm{~nm}$ red-shift and the intensity was enhanced dramatically. In the control systems, DSA-G $\subset$ H3-4C1P, DSA-G $\subset$ H4-2C1P, and individual DSA-G exhibited yellow emission centred at $\sim 530 \mathrm{~nm}$ without obvious wavelength shifts.

Furthermore, their detailed fluorescence behaviour along with control experiments have been investigated to test their supramolecular assembly-induced enhancement and FRET. H1-4C4P and H2-2C4P dissolved in chloroform exhibit weak blue fluorescence with emission maxima at $481 \mathrm{~nm}$ after excitation at $350 \mathrm{~nm}$. However, the yellow fluorescence at the emission maximum of $535 \mathrm{~nm}$ is strongly enhanced upon the gradual addition of DSA-G to the solution with the same excitation, while the emission at $481 \mathrm{~nm}$ simultaneously weakens (Fig. S19A and B, ESI $\dagger$ ). The fluorescent intensity of DSA-G $\subset \mathbf{H 2 - 2 C 4 P}$ is somewhat stronger than that of DSA-G $\subset$ H1-4C4P. The rapid enhancement in fluorescence can be observed until stoichiometric ratios are $1: 2$, after which the fluorescence intensity increases slowly. In comparison, the fluorescence spectra of DSA-G $\subset$ H3-4C1P (Fig. S19C, ESI $\dagger$ ), DSA-G $\subset$ H4-2C1P (Fig. S19D, ESI $\dagger$ ), DSA-G/ H5-4CM (Fig. S19E, ESI $\dagger$ ), DSA-G/H6-2CM (Fig. S19F, ESI $\dagger$ ), and individual DSA-G (Fig. S19G, ESI $\dagger$ ) have also been measured at the same concentrations as DSA-G, and there is no obvious fluorescence enhancement in the above systems except the linear weak increase due to the increased concentration of DSA-G. Thus, there are three major features contributing to the remarkable fluorescence enhancement of DSA-G $\subset$ H1-4C4P and DSA-G $\subset$ H2-2C4P (Fig. 1b): (i) FRET occurs between the two 

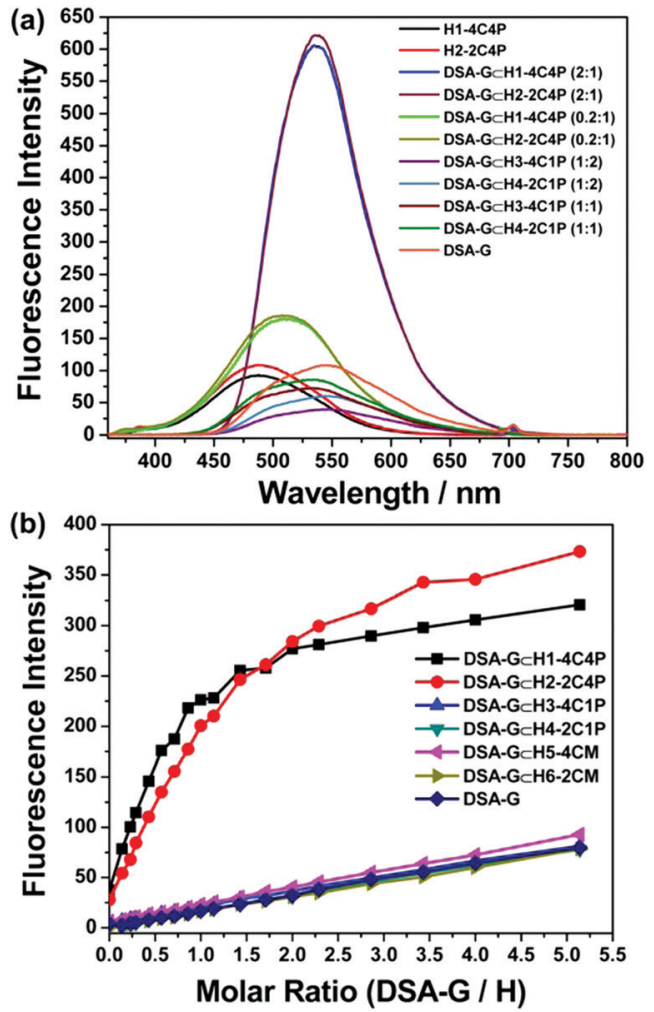

Fig. 1 (a) Fluorescence emission spectra $\left(\lambda_{\mathrm{ex}}=350 \mathrm{~nm}\right.$; slit widths: ex. $5 \mathrm{~nm}$, em. $5 \mathrm{~nm} ; 25^{\circ} \mathrm{C}$, [H1-4C4P] $=50 \mu \mathrm{M}$; [H2-4C4P] $=50 \mu \mathrm{M}$; [DSA$\mathbf{G} \subset \mathbf{H 1}-4 \mathbf{C} 4 \mathrm{P}]=100 \mu \mathrm{M} \subset 50 \mu \mathrm{M} ;[\mathrm{DSA}-\mathbf{G} \subset \mathbf{H 2}-2 \mathrm{C} 4 \mathrm{P}]=100 \mu \mathrm{M} \subset 50 \mu \mathrm{M}$; $[D S A-G \subset H 1-4 C 4 P]=10 \mu \mathrm{M} \subset 50 \mu \mathrm{M} ;[$ DSA-G $\subset$ H2-2C4P] $=10 \mu \mathrm{M} \subset$ $50 \mu \mathrm{M} ; \quad[\mathrm{DSA}-\mathrm{G} \subset \mathrm{H} 3-4 \mathrm{C} 1 \mathrm{P}]=100 \subset 200 \mu \mathrm{M} ; \quad[\mathrm{DSA}-\mathbf{G} \subset \mathbf{H} 4-2 \mathrm{C} 1 \mathrm{P}]=$

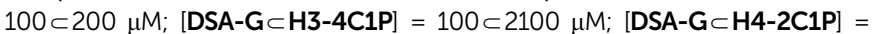
$100 \subset 100 \mu \mathrm{M} ;[\mathrm{DSA}-\mathrm{G}]=100 \mathrm{M})$ and $(\mathrm{b})$ changes of fluorescence emission intensity of DSA-G $\subset \mathrm{H} 1-4 \mathrm{C} 4 \mathrm{P}$, DSA-G $\subset \mathrm{H} 2-2 \mathrm{C} 4 \mathrm{P}$, DSA-G $\subset \mathrm{H} 3-4 \mathrm{C1P}$, DSA-G $\subset$ H4-2C1P, H5-4CM/DSA-G, H6-2CM/DSA-G and DSA-G (data gathered from Fig. S2O, ESI†).

AIEgens, i.e., TPE (donor) and DSA (acceptor), which is facilitated by the host-guest interaction; (ii) supramolecular assemblies bring TPE and DSA cores close to each other, resulting in the large restriction of internal rotation of AIE chromophores that populates the decay to the ground state; and (iii) the gradual addition of DSA-G. The increasing fluorescent intensity of DSAG $\subset$ H3-4C1P and DSA-G $\subset$ H4-2C1P is consistent with DSA-G, suggesting that the supramolecular assemblies contribute to the close distance between donors and acceptors.

Fluorescence quantum yields and fluorescence lifetimes have been measured using an integrating sphere and timeresolved fluorescence, respectively. The rate constants for radiative deactivation $\left(k_{\mathrm{r}}\right)$ and for non-radiative deactivation $\left(k_{\mathrm{nr}}\right)$ were elucidated. Compared with the control systems (DSAG $\subset$ H3-4C1P, DSA-G $\subset$ H4-2C1P, individual H1-4C4P, H2-2C4P and DSA-G), the ensembles of DSA-G $\subset$ H1-4C4P and DSA$\mathbf{G} \subset \mathbf{H 2}$-2C4P have higher quantum yields and $k_{\mathrm{r}}$, indicating that the supramolecular assembly and FRET contribute to the enhanced fluorescence synergistically (Table S1 and Fig. S20, S21, ESI $\dagger$ ). We calculated the energy transfer efficiency of DSAG $\subset$ H1-4C4P and DSA-G $\subset$ H2-2C4P both to be $88.6 \%$ through
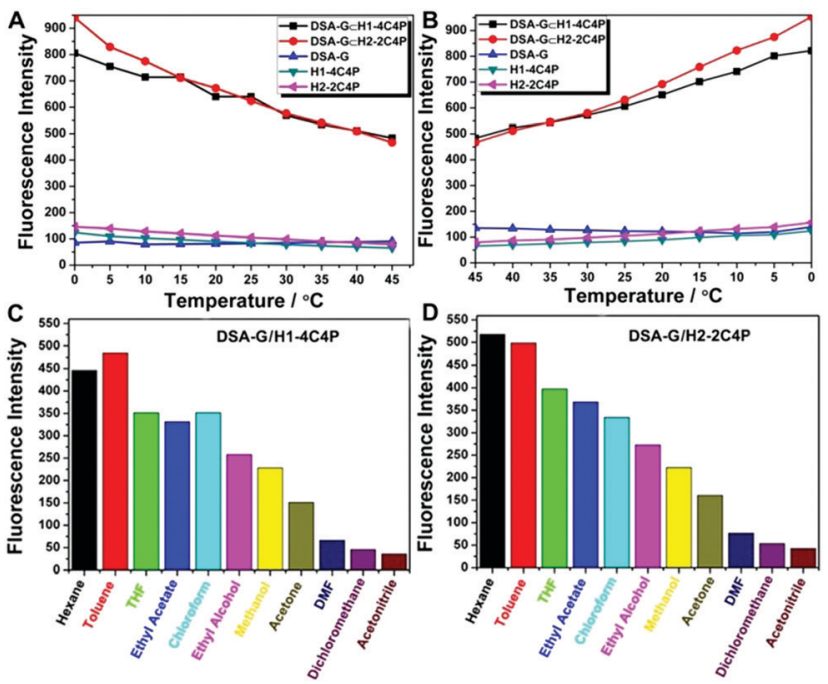

Fig. 2 Plots of fluorescence intensity changes of DSA-G $\subset$ H1-4C4P and DSA-G $\subset$ H2-2C4P (control experiments: individual H1-4C4P, H2-2C4P and DSA-G) at $\lambda_{\mathrm{em}}=535 \mathrm{~nm}$ upon (A) elevating and (B) lowering the temperature $\left(\lambda_{\mathrm{ex}}=350 \mathrm{~nm}\right.$; slit widths: ex. $3 \mathrm{~nm}$, em. $5 \mathrm{~nm} ; 2{ }^{\circ} \mathrm{C}$; concentrations: $[\mathrm{H} 1-4 \mathrm{C} 4 \mathrm{P}]=[\mathrm{H} 2-2 \mathrm{C} 4 \mathrm{P}]=35 \mu \mathrm{M},[\mathrm{DSA}-\mathrm{G}]=70 \mu \mathrm{M})$; histograms of fluorescent intensity changes of (C) DSA-G $\subset$ H1-4C4P and (D) DSA-G $\subset$ H2-2C4P influenced by solvents $\left(\lambda_{\mathrm{ex}}=350 \mathrm{~nm}\right.$; slit widths: ex. $3 \mathrm{~nm}$, em. $5 \mathrm{~nm} ; 25^{\circ} \mathrm{C}$; concentrations: $[\mathrm{H} 1-4 \mathrm{C} 4 \mathrm{P}]=[\mathrm{H} 2-2 \mathrm{C} 4 \mathrm{P}]=$ $14 \mu \mathrm{M},[\mathrm{DSA}-\mathrm{G}]=28 \mu \mathrm{M}$; all the solvents are mixed with $\mathrm{CHCl}_{3}$ at a volume ratio of $1: 1$ to guarantee the solubility).

the eqn (1), in which $I_{\mathrm{D}}$ stands for the fluorescence intensity of the donor without the acceptor while $I_{\mathrm{DA}}$ represents the intensity of the mixture of donor and acceptor.

$$
\phi_{\mathrm{ET}}=1-\frac{I_{\mathrm{D}}}{I_{\mathrm{DA}}}
$$

In the process of supramolecular assembly, the stabilities of host-guest interactions were always influenced by temperature changes because entropy governs their free energies of complexation. The fluorescence intensity of DSA-G $\subset$ H1-4C4P and DSA-G $\subset$ H2-2C4P was decreased upon gradually elevating the temperature from 0 to $45{ }^{\circ} \mathrm{C}$ (Fig. 2A, chloroform b.p.: $61.2^{\circ} \mathrm{C}$ ), while both increased upon lowering the temperature back to $0{ }^{\circ} \mathrm{C}$ (Fig. 2B and Fig. S23, ESI $\dagger$ ), indicating their thermo-responsive properties. In control experiments, individual H1-4C4P, H2-2C4P, and DSA-G at the same concentrations exhibited no obvious variations in fluorescence intensity upon changing the temperature (Fig. S22, ESI $\dagger$ ).

Considering the key roles of solvent composition in supramolecular assembly/disassembly, we also explored the influence of mixed solvents to the assemblies of DSA-G $\subset$ H1-4C4P and DSA$\mathbf{G} \subset \mathbf{H 2}-2 \mathbf{C 4 P}$ through changes in fluorescence emission intensity (Fig. 2C and D). Chloroform was chosen as the basic solvent to prepare the solutions of DSA-G $\subset$ H1-4C4P and DSA-G $\subset$ H2-2C4P (Fig. S24, ESI $\dagger$ ). As shown in Fig. 2D, the fluorescence intensities of DSA-G $\subset \mathbf{H 2 - 2 C 4 P}$ in the mixed solvents of hexane, toluene, $\mathrm{THF}$, and ethyl acetate with chloroform $(\mathrm{v} / \mathrm{v}=1: 1)$ were enhanced compared with that in pure chloroform, while the fluorescent intensities of DSA-G $\subset$ H1-4C4P were enhanced in mixed solvents 
of hexane/chloroform and toluene/chloroform. We ascribed their solvent-responsiveness to the cooperative impact of the polarities, viscosities, and solvent solubility, since the solubility of DSA-G $\subset$ H1-4C4P and DSA-G $\subset$ H2-2C4P and the polarities have significant influence on their molecular recognition events. On the basis of the above data, we conclude that the assembly of DSA-G $\subset \mathbf{H 2 - 2 C 4 P}$ exhibits relatively higher sensitivity toward both temperature and solvent composition as compared to that of DSA-G $\subset$ H1-4C4P, suggesting that the assembly of DSA-G $\subset \mathbf{H 2 - 2 C 4 P}$ has been affected more by the greater degree of restriction and relaxation of phenyl units in TPE cores led by the shorter lengths of carbon chains. Additional data including UV-Vis absorption spectra, quantum yields, and time-resolved fluorescence decay curves have also been obtained (Fig. S25-S28 and Tables S2, S3, ESI $\dagger$ ).

Photographs of solutions under natural light and $365 \mathrm{UV}$ lamp showed color changes with different stoichiometric ratios of guest and host, which are consistent with the results of the fluorescence spectra in Fig. 1. The color changes of the fluorescence can be attributed to the extent of energy transfer between the donor-acceptor pairs at different stoichiometries. As in Fig. 3, individual DSA-G molecules without donor molecules exhibit yellow fluorescence but are apparently dimmer than when the molecular ratio of DSA-G $\subset$ H1-4C4P is $2: 1$, which means the energy transfer from H1-4C4P plays a significant role. Yet when the stoichiometry is $0.2: 1$, part of the energy of the excited donor molecules has been released through emission, instead of being transferred to DSA-G. Partial energy transfer also manifests as shifts in the fluorescence emission spectra (Fig. 1).

Variable-temperature NMR analysis of DSA-G $\subset$ H1-4C4P and DSA-G $\subset$ H2-2C4P was performed (Fig. S29 and S30, ESI $\dagger$ ). The split peaks changed into broaden peak, indicating the formation of supramolecular architectures. The concentration-dependent DOSY

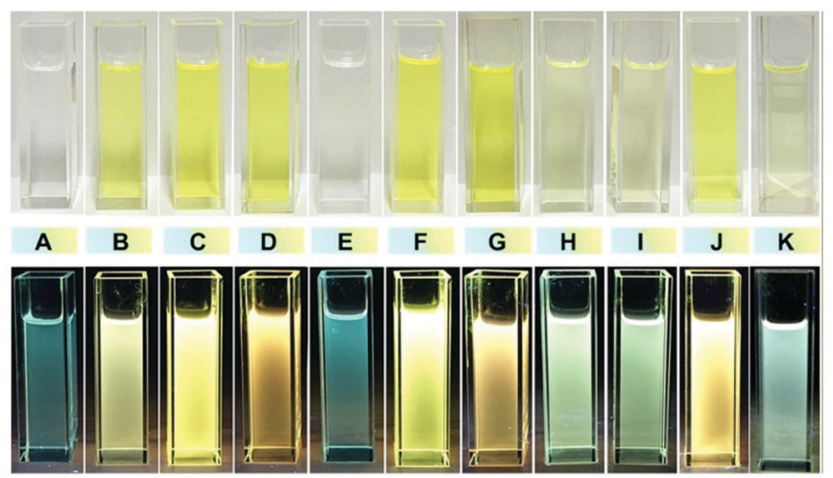

Fig. 3 Photographs (above) under natural light and (below) ultraviolet irradiation (lamp $\lambda_{\mathrm{em}}=365 \mathrm{~nm}$ ); (A) H1-4C4P ([H1-4C4P] $\left.=50 \mu \mathrm{M}\right)$; (B) DSA-G ([DSA-G] = $100 \mu \mathrm{M}) ;(\mathrm{C})$ DSA-G $\subset$ H1-4C4P $([\mathrm{H} 1-4 C 4 P]=$ $50 \mu \mathrm{M},[\mathrm{DSA}-\mathrm{G}]=100 \mu \mathrm{M}) ;(\mathrm{D}) \mathrm{DSA}-\mathrm{G} \subset \mathrm{H} 3-4 \mathrm{C} 1 \mathrm{P}([\mathrm{H3}-4 \mathrm{C} 1 \mathrm{P}]=200 \mu \mathrm{M}$, $[$ DSA-G] $=100 \mu \mathrm{M}) ;(\mathrm{E}) \mathrm{H2}-2 \mathrm{C} 4 \mathrm{P}([\mathrm{H} 2-4 \mathrm{C} 4 \mathrm{P}]=50 \mu \mathrm{M}) ;(\mathrm{F})$ DSA-G $\subset$ H2-2C4P $([\mathrm{H} 2-2 \mathrm{C} 4 \mathrm{P}]=50 \mu \mathrm{M},[\mathrm{DSA}-\mathrm{G}]=100 \mu \mathrm{M}) ;(\mathrm{G}) \mathbf{D S A}-\mathrm{G} \subset \mathrm{H} 4-2 \mathrm{C} 1 \mathrm{P}([\mathrm{H} 4-2 \mathrm{C} 1 \mathrm{P}]=$ $200 \mu \mathrm{M},[\mathrm{DSA}-\mathrm{G}]=100 \mu \mathrm{M}) ;(\mathrm{H})$ DSA-G $\subset$ H1-4C4P $([\mathrm{H} 1-4 \mathrm{C} 4 \mathrm{P}]=50 \mu \mathrm{M}$, $[\mathrm{DSA}-\mathrm{G}]=10 \mu \mathrm{M}) ;(\mathrm{I}) \mathrm{DSA}-\mathrm{G} \subset \mathrm{H} 2-2 \mathrm{C} 4 \mathrm{P}([\mathrm{H} 2-2 \mathrm{C} 4 \mathrm{P}]=50 \mu \mathrm{M}$, [DSA-G] $=$ $10 \mu \mathrm{M})$; (J) DSA-G $\subset$ H3-4C1P $([\mathrm{H3}-4 \mathrm{C} 1 \mathrm{P}]=100 \mu \mathrm{M}$, [DSA-G] $=100 \mu \mathrm{M})$; (K) DSA-G $\subset$ H4-2C1P $([\mathrm{H} 4-2 C 1 P]=100 \mu \mathrm{M}$, [DSA-G] $=100 \mu \mathrm{M})$

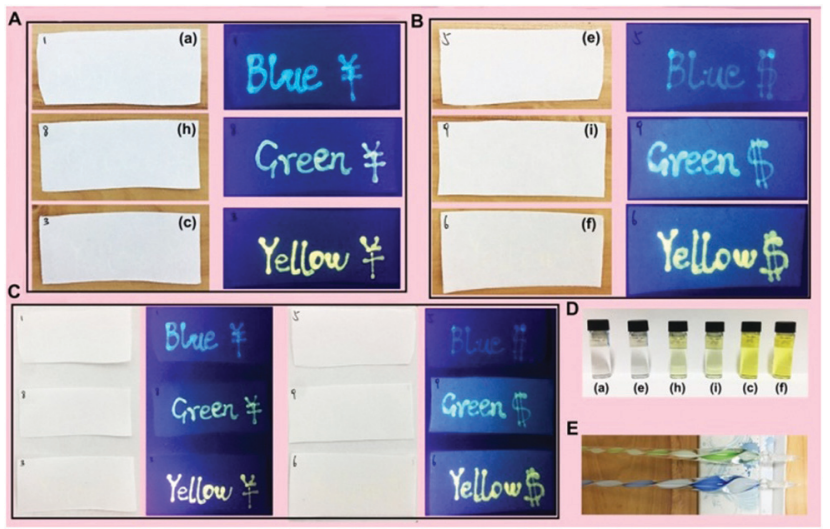

Fig. 4 Fluorescence intensity changes upon changes in temperature and solvents. (A) Application of DSA-G $\subset \mathbf{H 1}-\mathbf{4 C} \mathbf{C P}(\mathrm{a}, \mathrm{h}, \mathrm{c})$ and (B) DSA-G $\subset$ H22C4P (e, i, f) written on filter paper, (left: under natural light, right: under ultraviolet light, $\lambda_{\mathrm{em}}=365 \mathrm{~nm}$ ) as tunable fluorescent inks. (C) Fluorescent inks of DSA-G $\subset \mathbf{H 1}-\mathbf{4 C 4 P}(\mathrm{a}, \mathrm{h}, \mathrm{c})$ and DSA-G $\subset \mathbf{H 2 - 2 C 4 P}(\mathrm{e}, \mathrm{i}, \mathrm{f})$ on the same white paper; (a: H1-4C4P $([\mathbf{H 1}-4 C 4 P]=50 \mu \mathrm{M})$; $\mathrm{h}: \mathbf{D S A}-\mathbf{G} \subset \mathbf{H 1}-$ 4C4P $([\mathrm{H} 1-4 C 4 P]=50 \mu \mathrm{M},[\mathrm{DSA}-\mathrm{G}]=10 \mu \mathrm{M}) ; \mathrm{C}:$ DSA-G $\subset$ H1-4C4P $([\mathrm{H} 1-$ 4C4P $]=50 \mu \mathrm{M},[$ DSA-G] $=100 \mu \mathrm{M}) ; \mathrm{e}: \mathrm{H2}-2 \mathrm{C} 4 \mathrm{P}([\mathrm{H2}-4 \mathrm{C} 4 \mathrm{P}]=50 \mu \mathrm{M})$; i: DSA-G $\subset$ H2-2C4P $([\mathbf{H 2}-2 C 4 P]=50 \mu \mathrm{M}$, [DSA-G] $=10 \mu \mathrm{M}) ;$ f: DSA-G $\subset$ H2-2C4P $([\mathrm{H} 2-2 \mathrm{C} 4 \mathrm{P}]=50 \mu \mathrm{M},[\mathrm{DSA}-\mathrm{G}]=100 \mu \mathrm{M}))$. Images of $(\mathrm{D})$ the solutions and (E) solution-loaded pens.

spectra (Fig. S31 and S32, ESI $\dagger$ ) of DSA-G $\subset$ H1-4C4P and DSA$\mathbf{G} \subset \mathbf{H 2}$-2C4P indicated the changes of their diffusion coefficients. Compared with individual DSA-G with the diffusion coefficient of $(2.87 \pm 0.08) \times 10^{-9} \mathrm{~m}^{2} \mathrm{~s}^{-1}$, the diffusion coefficient decreased upon addition of H1-4C4P or H2-2C4P, suggesting that they gradually assembled into large polymeric architectures.

Similar color changes can also be observed in fluorescence microscopy images, which indicate their solid-state fluorescent properties (Fig. S33-S36, ESI $\dagger$ ). CIE coordinates showed in Fig. S37 (ESI $\dagger$ ) also confirmed the color changes as blue, green, and yellow of the supramolecular ensembles (i.e., DSA-G $\subset$ H1-4C4P, DSA-G $\subset$ H2-2C4P, and individual DSA-G, respectively).

In order to determine the solid-state fluorescence emission, measurements of fluorescent inks have also been performed (Fig. 4). Considering that chloroform can make plastics swell, the solutions were loaded into glass pens and written on filter paper (Fig. 4E). Similarly, the colours of the characters also changed from blue to green, and then to yellow upon the gradual addition of DSA-G. The handwriting on the coated papers lasted for at least $24 \mathrm{~h}$, indicating that the tunable fluorescence of DSA-G $\subset$ H1-4C4P and DSA-G $\subset$ H2-2C4P were the same and efficient both in solution and the solid state.

\section{Conclusions}

In summary, tunable solid-state fluorescent materials were fabricated through supramolecular assembly of DSA-G $\subset$ H1-4C4P and DSA-G $\subset$ H2-2C4P, accompanied by supramolecular assemblyinduced emission enhancement and FRET facilitated by molecular recognition. The remarkable fluorescence emission enhancement and the tunable fluorescence, ranges from blue to green to yellow, resulting from different stoichiometric ratios of DSA-G. Both of 
the ensembles of DSA-G $\subset$ H1-4C4P and DSA-G $\subset$ H2-2C4P exhibited thermo- and solvent-responsive properties, showing great potential for smart optical switches. DSA-G $\subset$ H2-2C4P possesses relative higher sensitivity to external stimuli compared with DSA-G $\subset$ H14C4P. Furthermore, fluorescence microscopy images and experiments on fluorescent inks of these assemblies confirmed that their tunable fluorescence emission was efficient, even in the solid state. These tunable solid fluorescent materials possess great potential in smart optical devices. Efforts are ongoing in our laboratory to develop tunable solid-state fluorescent materials with extended wavelengths, taking advantage of supramolecular assembly and molecular machinery, to endow them with reversible stimuli-responsiveness.

\section{Conflicts of interest}

There are no conflicts to declare.

\section{Acknowledgements}

This work was supported by the National Natural Science Foundation of China (51673084), the Jilin Province University Cooperative Construction Project - Special Funds for New Materials (SXGJSF2017-3), and the Jilin University Talents Cultivation Program. N. S. thanks the support by the scholarship from China Scholarship Council (CSC).

\section{Notes and references}

1 M. Zhu and C. Yang, Blue fluorescent emitters: design tactics and applications in organic light-emitting diodes, Chem. Soc. Rev., 2013, 42, 4963-4976.

2 X. Hou, C. Ke, C. J. Bruns, P. R. McGonigal, R. B. Pettman and J. F. Stoddart, Tunable solid-state fluorescent materials for supramolecular encryption, Nat. Commun., 2015, 6, 6884.

3 X.-L. Ni, S. Chen, Y. Yang and Z. Tao, Facile cucurbit[8]urilbased supramolecular approach to fabricate tunable luminescent materials in aqueous solution, J. Am. Chem. Soc., 2016, 138, 6177-6183.

4 Y. Hong, J. W. Y. Lam and B. Z. Tang, Aggregation-induced emission, Chem. Soc. Rev., 2011, 40, 5361-5388.

5 H. Zhang, J. Liu, L. Du, C. Ma, N. L. C. Leung, Y. Niu, A. Qin, J. Sun, Q. Peng, H. H. Y. Sung, I. D. Williams, R. T. K. Kwok, J. W. Y. Lam, K. S. Wong, D. L. Phillips and B. Z. Tang, Drawing a clear mechanistic picture for the aggregationinduced emission process, Mater. Chem. Front., 2019, 3, 1143-1150.

6 P. A. A. M. Vaz, J. Rocha, A. M. S. Silva and S. Guieu, Aggregation-induced emission enhancement of chiral boranils, New J. Chem., 2018, 42, 18166-18171.

7 J. Luo, Z. Xie, J. W. Y. Lam, L. Cheng, H. Chen, C. Qiu, H. S. Kwok, X. Zhan, Y. Liu, D. Zhu and B. Z. Tang, Aggregationinduced emission of 1-methyl-1,2,3,4,5-pentaphenylsilole, Chem. Commun., 2001, 1740-1741.
8 S. Chen, Y. Hong, Y. Liu, J. Liu, C. Leung, M. Li, R. T. K. Kwok, E. Zhao, J. W. Y. Lam, Y. Yu and B. Z. Tang, Full-range intracellular $\mathrm{pH}$ sensing by an aggregation-induced emissionactive two-channel ratiometric fluorogen, J. Am. Chem. Soc., 2013, 135, 4926-4929.

9 C. W. T. Leung, Y. Hong, S. Chen, E. Zhao, J. W. Y. Lam and B. Z. Tang, A photostable AIE luminogen for specific mitochondrial imaging and tracking, J. Am. Chem. Soc., 2013, 135, 62-65.

10 H. Nie, K. Hu, Y. Cai, Q. Peng, Z. Zhao, R. Hu, J. Chen, S.-J. $\mathrm{Su}$, A. Qin and B. Z. Tang, Tetraphenylfuran: aggregationinduced emission or aggregation-caused quenching, Mater. Chem. Front., 2017, 1, 1125-1129.

11 X. Yan, T. R. Cook, P. Wang, F. Huang and P. J. Stang, Highly emissive platinum(II) metallacages, Nat. Chem., 2015, 7, 342-348.

12 Q.-W. Zhang, D. Li, X. Li, P. B. White, J. Mecinović, X. Ma, H. Ågren, R. J. M. Nolte and H. Tian, Multicolor photoluminescence including white-light emission by a single hostguest complex, J. Am. Chem. Soc., 2016, 138, 13541-13550.

13 C. Fan, W. Wu, J. J. Chruma, J. Zhao and C. Yang, Enhanced triplet-triplet energy transfer and upconversion fluorescence through host-guest complexation, J. Am. Chem. Soc., 2016, 138, 15405-15412.

14 B. Shi, K. Jie, Y. Zhou, J. Zhou, D. Xia and F. Huang, Nanoparticles with near-infrared emission enhanced by pillararene-based molecular recognition in water, J. Am. Chem. Soc., 2016, 138, 80-83.

15 K. Wang, C.-Y. Wang, Y. Zhang, S. X.-A. Zhang, B. Yang and Y.-W. Yang, Ditopic pillar[5] arene-based fluorescence enhancement material mediated by [c2] daisy chain formation, Chem. Commun., 2014, 50, 9458-9461.

16 T. Ogoshi, S. Kanai, S. Fujinami, T. Yamagishi and Y. Nakamoto, para-Bridged symmetrical pillar[5]arenes: their Lewis acid catalyzed synthesis and host-guest property, J. Am. Chem. Soc., 2008, 130, 5022-5023.

17 N. Song, T. Kakuta, T.-A. Yamagishi, Y.-W. Yang and T. Ogoshi, Molecular scale porous materials based on pillar[n]arenes, Chem, 2018, 4, 2029-2053.

18 M. Xue, Y. Yang, X. Chi, Z. Zhang and F. Huang, Pillararenes, a new class of macrocycles for supramolecular chemistry, Acc. Chem. Res., 2012, 45, 1294-1308.

19 N. Strutt, L. H. Zhang, S. T. Schneebeli and J. F. Stoddart, Functionalizing pillar[n]arenes, Acc. Chem. Res., 2014, 47, 2631-2642.

20 Y.-W. Yang, Y.-L. Sun and N. Song, Switchable host-guest systems on surfaces, Acc. Chem. Res., 2014, 47, 1950-1960.

21 D. Cao, Y. Kou, J. Liang, Z. Chen, L. Wang and H. Meier, A facile and efficient preparation of pillararenes and a pillarquinone, Angew. Chem., Int. Ed., 2009, 48, 9721-9723.

22 J. Han, X. Hou, C. Ke, H. Zhang, N. L. Strutt, C. L. Stern and J. F. Stoddart, Activation-enabled syntheses of functionalized pillar[5]arene derivatives, Org. Lett., 2015, 17, 3260-3263.

23 W.-B. Hu, W.-J. Hu, Y. A. Liu, J.-S. Li, B. Jiang and K. Wen, Negative cooperativity in the binding of imidazolium and viologen ions to a pillar[5] arene-crown ether fused host, $\mathrm{Org}$. Lett. , 2015, 17, 2940-2943. 
24 K. Wang, L.-L. Tan, D.-X. Chen, N. Song, G. Xi, S. X.-A. Zhang, C. Li and Y.-W. Yang, One-pot synthesis of pillar[n]arenes catalyzed by a minimum amount of $\mathrm{TfOH}$ and a solutionphase mechanistic study, Org. Biomol. Chem., 2012, 10, 9405-9409.

25 X. Wang, X.-Y. Lou, X.-Y. Jin, F. Liang and Y.-W. Yang, A binary supramolecular assembly with intense fluorescence emission, high $\mathrm{pH}$ stability, and cation selectivity: supramolecular assembly-induced emission materials, Research, 2019, 1454562.

26 H. Zhang, Z. Liu and Y. Zhao, Pillararene-based self-assembled amphiphiles, Chem. Soc. Rev., 2018, 47, 5491-5528.

27 B. Hua, W. Zhou, Z. Yang, Z. Zhang, L. Shao, H. Zhu and F. Huang, Supramolecular solid-state microlaser constructed from pillar[5]arene-based host-guest complex microcrystals, J. Am. Chem. Soc., 2018, 140, 15651-15654.

28 Y.-F. Li, Z. Li, Q. Lin and Y.-W. Yang, Functional supramolecular gels based on pillar[n]arene macrocycles, Nanoscale, 2020, 12, 2180-2200.

29 C.-F. Zhang, S.-H. Li, C.-C. Zhang and Y. Liu, Molecular binding behaviors and thermodynamics of ferrocenyl dimethylaminium derivatives by anionic pillar[5]arene, Org. Biomol. Chem., 2015, 13, 10808-10812.

30 L. Jiang, X. Huang, D. Chen, H. Yan, X. Li and X. Du, Supramolecular vesicles coassembled from disulfide-linked benzimidazolium amphiphiles and carboxylate-substituted pillar[6]arenes that are responsive to five stimuli, Angew. Chem., Int. Ed., 2017, 56, 2655-2659.

31 X. Liao, L. Guo, J. Chang, S. Liu, M. Xie and G. Chen, Thermoresponsive AuNPs stabilized by pillararene-containing polymers, Macromol. Rapid Commun., 2015, 36, 1492-1497.

32 N. L. Strutt, D. Fairen-Jimenez, J. Iehl, M. B. Lalonde, R. Q. Snurr, O. K. Farha, J. T. Hupp and J. F. Stoddart, Incorporation of an A1/A2-difunctionalized pillar[5] arene into a metal-organic framework, J. Am. Chem. Soc., 2012, 134, 17436-17439.

33 Z. Li, N. Song and Y.-W. Yang, Stimuli-responsive drug delivery systems based on supramolecular nanovalves, Matter, 2019, 1, 345-368.

34 N. Song and Y.-W. Yang, Molecular and supramolecular switches on mesoporous silica nanoparticles, Chem. Soc. Rev., 2015, 44, 3474-3504.

35 L.-L. Tan, H. Li, Y.-C. Qiu, D.-X. Chen, X. Wang, R.-Y. Pan, Y. Wang, S. X.-A. Zhang, B. Wang and Y.-W. Yang, Stimuliresponsive metal-organic frameworks gated by pillar[5]arene supramolecular switches, Chem. Sci., 2015, 6, 1640-1644.

36 Y. Cao, X.-Y. Hu, Y. Li, X. Zou, S. Xiong, C. Lin, Y.-Z. Shen and L. Wang, Multistimuli-responsive supramolecular vesicles based on water-soluble pillar[6]arene and SAINT complexation for controllable drug release, J. Am. Chem. Soc., 2014, 136, 10762-10769.

37 Q.-L. Li, Y. Sun, L. Ren, X. Wang, C. Wang, L. Li, Y.-W. Yang, $\mathrm{X}$. Yu and J. Yu, Supramolecular nanosystem based on pillararene-capped CuS nanoparticles for targeted chemophotothermal therapy, ACS Appl. Mater. Interfaces, 2018, 10, 29314-29324.
38 J. Yang, D. Dai, X. Lou, L. Ma, B. Wang and Y.-W. Yang, Supramolecular nanomaterials based on hollow mesoporous drug carriers and macrocycle-capped $\mathrm{CuS}$ nanogates for synergistic chemo-photothermal therapy, Theranostics, 2020, 10, 615-629.

39 X.-B. Hu, Z. Chen, G. Tang, J.-L. Hou and Z.-T. Li, Singlemolecular artificial transmembrane water channels, J. Am. Chem. Soc., 2012, 134, 8384-8387.

40 W. Si, Z.-T. Li and J.-L. Hou, Voltage-driven reversible insertion into and leaving from a lipid bilayer: tuning transmembrane transport of artificial channels, Angew. Chem., Int. Ed., 2014, 53, 4578-4581.

41 L. Chen, W. Si, L. Zhang, G. Tang, Z.-T. Li and J.-L. Hou, Chiral selective transmembrane transport of amino acids through artificial channels, J. Am. Chem. Soc., 2013, 135, 2152-2155.

42 G. Yu, J. Zhou, J. Shen, G. Tang and F. Huang, Cationic pillar[6]arene/ATP host-guest recognition: selectivity, inhibition of ATP hydrolysis, and application in multidrug resistance treatment, Chem. Sci., 2016, 7, 4073-4078.

43 D.-X. Chen, Y.-L. Sun, Y. Zhang, J.-Y. Cui, F.-Z. Shen and Y.-W. Yang, Supramolecular self-assembly and photophysical properties of pillar[5]arene-stabilized CdTe quantum dots mediated by viologens, RSC Adv., 2013, 3, 5765-5768.

44 H. Li, D.-X. Chen, Y.-L. Sun, Y. B. Zheng, L.-L. Tan, P. S. Weiss and Y.-W. Yang, Viologen-mediated assembly of and sensing with carboxylatopillar[5] arene-modified gold nanoparticles, J. Am. Chem. Soc., 2013, 135, 1570-1576.

45 X. Wang, Z.-J. Liu, E. H. Hill, Y. Zheng, G. Guo, Y. Wang, P. S. Weiss, J. Yu and Y.-W. Yang, Organic-inorganic hybrid pillarene-based nanomaterial for label-free detection and catalysis, Matter, 2019, 1, 848-861.

46 X. Mao, T. Liu, J. Bi, L. Luo, D. Tian and H. Li, The synthesis of pillar[5]arene functionalized graphene as a fluorescent probe for paraquat in living cells and mice, Chem. Commun., 2016, 52, 4385-4388.

47 T. Ogoshi, D. Yamafuji, L. T. Yamagishi and A. M. Brouwer, Förster resonance energy transfer by formation of a mechanically interlocked [2]rotaxane, Chem. Commun., 2013, 49, 5468-5470.

48 J. Gao, M.-X. Wu, D. Dai, Z. Cai, Y. Wang, W. Fang, Y. Wang and Y.-W. Yang, N-doped carbon dots covalently functionalized with pillar[5]arenes for $\mathrm{Fe}^{3+}$ sensing, Beilstein J. Org. Chem., 2019, 15, 1262-1267.

49 X. Chi, X. Ji, D. Xia and F. Huang, A dual-responsive supraamphiphilic polypseudorotaxane constructed from a watersoluble pillar[7] arene and an azobenzene-containing random copolymer, J. Am. Chem. Soc., 2015, 137, 1440-1443.

50 K. Jie, Y. Zhou, Y. Yao, B. Shi and F. Huang, $\mathrm{CO}_{2}$-responsive pillar[5]arene-based molecular recognition in water: establishment and application in gas-controlled self-assembly and release, J. Am. Chem. Soc., 2015, 137, 10472-10475.

51 J. Yang, G. Yu, D. Xia and F. Huang, A pillar[6]arene-based UV-responsive supra-amphiphile: synthesis, self-assembly, and application in dispersion of multiwalled carbon nanotubes in water, Chem. Commun., 2014, 50, 3993-3995. 
52 M.-X. Wu and Y.-W. Yang, Metal-organic framework (MOF)based drug/cargo delivery and cancer therapy, Adv. Mater., 2017, 29, 1606134.

53 K. Wang, C.-Y. Wang, Y. Wang, H. Li, C.-Y. Bao, J.-Y. Liu, S. X.-A. Zhang and Y.-W. Yang, Electrospun nanofibers and multi-responsive supramolecular assemblies constructed from a pillar[5]arene-based receptor, Chem. Commun., 2013, 49, 10528-10530.

54 Z.-Y. Li, Y. Zhang, C.-W. Zhang, L.-J. Chen, C. Wang, H. Tan, Y. Yu, X. Li and H.-B. Yang, Cross-linked supramolecular polymer gels constructed from discrete multi-pillar[5]arene metallacycles and their multiple stimuli-responsive behavior, J. Am. Chem. Soc., 2014, 136, 8577-8589.

55 C.-L. Sun, J.-F. Xu, Y.-Z. Chen, L.-Y. Niu, L.-Z. Wu, C.-H. Tung and Q.-Z. Yang, Synthesis of a disulfide-bridged bispillar[5] arene and its application in supramolecular polymers, Polym. Chem., 2016, 7, 2057-2061.

56 S.-Y. Zhou, N. Song, S.-X. Liu, D.-X. Chen, Q. Jia and Y.-W. Yang, Separation and preconcentration of gold and palladium ions with a carboxylated pillar[5]arene derived sorbent prior to their determination by flow injection FAAS, Microchim. Acta, 2014, 181, 1551-1556.

57 T. Zhou, N. Song, H. Yu and Y.-W. Yang, Pillar[5,6]arenefunctionalized silicon dioxide: synthesis, characterization, and adsorption of herbicide, Langmuir, 2015, 31, 1454-1461.

58 L.-L. Tan, H. Li, Y. Tao, S. X.-A. Zhang, B. Wang and Y.-W. Yang, Pillar[5]arene-based supramolecular organic frameworks for highly selective $\mathrm{CO}_{2}$-capture at ambient conditions, Adv. Mater., 2014, 26, 7027-7031.

59 Y. Fang, X. Yuan, L. Wu, Z. Peng, W. Feng, N. Liu, D. Xu, S. Li, A. Sengupta, P. K. Mohapatra and L. Yuan, Ditopic CMPO-pillar[5]arenes as unique receptors for efficient separation of americium(III) and europium(III), Chem. Commun., 2015, 51, 4263-4266.

60 H. Zhang, X. Ma, K. T. Nguyen and Y. Zhao, Biocompatible pillararene-assembly-based carriers for dual bioimaging, ACS Nano, 2013, 7, 7853-7863.

61 Y. Chang, K. Yang, P. Wei, S. Huang, Y. Pei, W. Zhao and Z. Pei, Cationic vesicles based on amphiphilic pillar[5]arene capped with ferrocenium: a redox-responsive system for drug/siRNA co-delivery, Angew. Chem., Int. Ed., 2014, 126, 13342-13346.

62 C. Sathiyajith, R. R. Shaikh, Q. Han, Y. Zhang, K. Meguellati and Y.-W. Yang, Biological and related applications of pillar[n]arenes, Chem. Commun., 2017, 53, 677-696.

63 N. Song, X.-Y. Lou, L. Ma, H. Gao and Y.-W. Yang, Supramolecular nanotheranostics based on pillarenes, Theranostics, 2019, 9, 3075-3093.

64 D.-D. Zheng, D.-Y. Fu, Y. Wu, Y.-L. Sun, L.-L. Tan, T. Zhou, S.-Q. Ma, X. Zha and Y.-W. Yang, Efficient inhibition of human papillomavirus $16 \mathrm{~L} 1$ pentamer formation by a carboxylatopillarene and a $p$-sulfonatocalixarene, Chem. Commun., 2014, 50, 3201-3203.
65 S. Yan, S. Chen, X. Gou, J. Yang, J. An, X. Jin, Y.-W. Yang, L. Chen and H. Gao, Biodegradable supramolecular materials based on cationic polyaspartamides and pillar[5]arene for targeting Gram-positive bacteria and mitigating antimicrobial resistance, Adv. Funct. Mater., 2019, 29, 1904683.

66 N. Song, D.-X. Chen, Y.-C. Qiu, X.-Y. Yang, B. Xu, W. Tian and Y.-W. Yang, Stimuli-responsive blue fluorescent supramolecular polymers based on a pillar[5] arene tetramer, Chem. Commun., 2014, 50, 8231-8234.

67 N. Song, X.-Y. Lou, W. Hou, C.-Y. Wang, Y. Wang and Y.-W. Yang, Pillararene-based fluorescent supramolecular systems: the key role of chain length in gelation, Macromol. Rapid Commun., 2018, 39, 1800593.

68 X.-H. Wang, N. Song, W. Hou, C.-Y. Wang, Y. Wang, J. Tang and Y.-W. Yang, Efficient aggregation-induced emission manipulated by polymer host materials, Adv. Mater., 2019, 31, 1903962.

69 N. Song, D.-X. Chen, M.-C. Xia, X.-L. Qiu, K. Ma, B. Xu, W. Tian and Y.-W. Yang, Supramolecular assembly-induced yellow emission of 9,10-distyrylanthracene bridged bis(pillar[5]arene)s, Chem. Commun., 2015, 51, 5526-5529.

70 X.-Y. Lou, N. Song and Y.-W. Yang, Enhanced solution and solid-state emission and tunable white-light emission harvested by supramolecular approaches, Chem. - Eur. J., 2019, 25, 11975-11982.

71 X.-Y. Lou and Y.-W. Yang, Manipulating aggregationinduced emission with supramolecular macrocycles, Adv. Opt. Mater., 2018, 6, 1800668.

72 J.-R. Wu and Y.-W. Yang, New opportunities in synthetic macrocyclic arenes, Chem. Commun., 2019, 55, 1533-1543.

73 J. Wu, S. Sun, X. Feng, J. Shi, X.-Y. Hu and L. Wang, Controllable aggregation-induced emission based on a tetraphenylethylene-functionalized pillar[5] arene via host-guest recognition, Chem. Commun., 2014, 50, 9122-9125.

74 R. Chen, H. Jiang, H. Gu, Q. Zhou, J. Wu, D. Chen and J. Zhang, A pH-responsive fluorescent [5]pseudorotaxane formed by self-assembly of cationic water-soluble pillar[5]arenes and a tetraphenylethene derivative, Chem. Commun., 2015, 51, 12220-12223.

75 C. Liu, H. Yu, Q. Li, C. Zhu and Y. Xia, Brighter, more stable, and less toxic: a host-guest interaction-aided strategy for fabricating fluorescent silica nanoparticles and applying them in bioimaging and biosensing at the cellular level, ACS Appl. Mater. Interfaces, 2018, 10, 16291-16298.

76 B. Han, L. Zhu, X. Wang, M. Bai and J. Jiang, Conformationcontrolled emission of AIE luminogen: a tetraphenylethene embedded pillar[5]arene skeleton, Chem. Commun., 2018, 54, 837-840.

77 C.-L. Sun, H.-Q. Peng, L.-Y. Niu, Y.-Z. Chen, L.-Z. Wu, C.-H. Tung and Q.-Z. Yang, Artificial light-harvesting supramolecular polymeric nanoparticles formed by pillar[5] arene-based host-guest interaction, Chem. Commun., 2018, 54, 1117-1120. 